\title{
The impact of Brazilian samba on balance and quality of life of individuals with Parkinson's disease
}

\section{O impacto do samba brasileiro no equilíbrio e na qualidade de vida de indivíduos com doença de Parkinson}

\section{AUTHOR'S \\ Líria Cruz Fonseca ${ }^{1}$ (D) \\ Ana Cristina Tillmann ${ }^{1}$ (D) \\ Jéssica Moratelli ${ }^{1}$ (D) \\ Alessandra Swarowsky ${ }^{2}$ (DC \\ Adriana Coutinho de Azevedo Guimarães ${ }^{1}$ (iD \\ 1 University of the State of Santa Catarina, Physical Education Department, Florianópolis, Santa Catarina, Brazil. \\ 2 University of the State of Santa Catarina, Physiotherapy Department, Florianópolis, Santa Catarina, Brazil.}

\section{CORRESPONDING}

Jéssica Moratelli

jessica.moratelli@hotmail.com

Adress: Juan Carlos Manucci, 88, Kobrasol, São José, Santa Catarina.

Zip code: 88080-350

DOI

$10.12820 /$ rbafs.26e0194

\section{(cc) BY}

This work is licensed under a Creative Commons Attribution 4.0 International License.

\begin{abstract}
This non-randomized clinical trial aimed to analyze the impact of the Brazilian samba training protocol on the balance and quality of life of people with Parkinson's disease. Forty-seven individuals participated, with a mean age of $68 \pm 9.3$ years-old, 24 from the control group (CG) and 23 from the experimental group (EG). The CG was formed by those who did not participate in the intervention, and the EG by individuals who participated in the Brazilian samba dance protocol. This study was divided into pre-intervention (before 12 weeks) and post-intervention (after 12 weeks) with a questionnaire consisting of: Mini Mental State Examination (MMSE); Unified Parkinson's Disease Rating Scale (UPDRS); Quality of life (PDQ-39); Berg's Balance Scale; Perception of perceived changes. The results point to a significant improvement after the intervention in the UPDRS ( $p<$ $0.001)$ and balance ( $p=0.006)$ of the EG; in the quality of life of the EG after intervention in the mobility ( $p=0.009)$ and total $(p=0.034)$ domains; and in the post-intervention period in the cognitive $(p=0.025)$ and communication $(p=0.032)$ domains of the EG and CG. Thus, it is concluded that the Brazilian samba rhythm has been shown to be effective in improving the total UPDRS, balance and quality of life, as well as in mobility, cognition and communication.
\end{abstract}

Keywords: Parkinson's disease; Balance; Quality of life; Samba.

RESUMO

Esse ensaio clínico não randomizado teve como objetivo analisar o impacto do protocolo de treinamento de samba brasileiro no equilíbrio e na qualidade de vida de pessoas com a doença de Parkinson. Participaram quarenta e sete individuos, média de idade de $68 \pm 9,3$ anos, 24 do grupo controle (GC) e 23 do grupo experimental (GE). O GC foi formado por aqueles que não participaram da intervenção, e o GE por indivíduos que participaram do protocolo de dança do samba brasileiro. Este estudo foi dividido em pré-intervenção (antes das 12 semanas) e pós-intervenção (após as 12 semanas) com um questionário composto por: Mini Exame do Estado Mental (MEEM); Escala Unificada de Avaliação de Doença de Parkinson (UPDRS); Qualidade de vida (PDQ-39); Escala de Equilíbrio de Berg; Percepção das mudanças percebidas. Os resultados apontam para uma melhora significativa após a intervenção no UPDRS $(p<0,001)$ e equilíbrio $(p=$ $0,006)$ do GE; na qualidade de vida do GE pós intervenção nos dominios mobilidade $(p=0,009)$ e total $(p$ $=0,034)$; e no periodo pós intervenção nos domínios cognitivos $(p=0,025)$ e comunicação $(p=0,032)$ do $G E$ e GC. Desta forma conclui-se que o ritmo samba brasileiro tem se mostrado eficaz na melhora do UPDRS total, do equilíbrio e da qualidade de vida, como na mobilidade, cognição e comunicação.

Palavras-chave: Doença de parkinson; Equilíbrio; Qualidade de vida; Samba.

\section{Introduction}

Deficits in functional balance are among the most prevalent symptoms in Parkinson's disease (PD), present in $38-68 \%$ of individuals who live with the disease. The loss of balance may be one of the main factors responsible for the increase in the number of falls in individuals with PD and are identified as one of the most critical consequences of the disease ${ }^{1}$. Thus, in addition to altering their ability to act, their confidence decreases, increasing the fear of possible falls and reducing the practice of so- cial or even daily activities and leisure, and consequently worsens the quality of life and mental health ${ }^{2}$.

Regular and supervised balance training is highly recommended to maintain physical performance in $\mathrm{PD}$, in this context, activities such as Tai Chi, multimodal training, hydrotherapy and physiotherapy have resulted in positive results, with improved functional balance of individuals ${ }^{3,4}$. In addition to dance, studies have shown that Ballet, Tango Argentine and ballroom dancing can promote physical and psychosocial bene- 
fits to the practicing individuals, as well as better scores in the balance tests applied after the interventions ${ }^{5}$.

The dance encourages hearing, cognitive strategies, balance exercises and physical activity in a pleasurable approach, due to the interaction with music and movement, facilitating their adherence to the activity ${ }^{6}$. Moreover, it allows for interpersonal relationships, reducing social isolation and improving quality of life. According to Sharp \& Hewit ${ }^{7}$, dance therapy results in significant motor exploration enhancements of the Unified Parkinson's Disease Assessment Scale (UPDRS), balance and gait speed.

Thus, it is important to consider an individual's personal, cultural and social preferences to promote rehabilitation program's adherence ${ }^{8}$. In view of these preferences, Brazilian samba seems to serve not only the Brazilian population, as it is a culturally known rhythm in Brazil but it is also practiced worldwide, in addition to being a quaternary rhythm just like Tango, which is the most investigated by individuals with $\mathrm{PD}^{9}$. Therefore, this study aimed to analyze the impact of the Brazilian samba training protocol on the balance and quality of life of individuals with PD.

\section{Methods}

This was a non-randomized clinical trial with 47 individuals diagnosed with PD $(68.3 \pm 9.3$ years $)$. The recruitment was conducted through telephone calls with the invitation to participate voluntarily in dance and physical therapy classes; those who did not accept to participate in the dance intervention were included in the control group $(\mathrm{CG})(67.6 \pm 10.9$ years $)$ and to perform only physiotherapy and those who accepted were included in the experimental group (EG) $(65.30$ \pm 10.5 years) and included in the Extension Program Rhythm and Movement at Santa Catarina State University (UDESC) performing the classes at Catarinense Rehabilitation Center (CCR) in Florianópolis, Santa Catarina. Figure 1 displays the selection criteria of study participants.

The study included individuals with a disease's medical diagnosis according to the criteria of the London Brain Bank ${ }^{10}$; with drug therapy stabilized - leastways - four months; in the on-medication stage; of both sexes; age $\geq 40$ years, attending physical therapy at least once a week and had not performed any type of dance within the last three months prior to the study; a score of 1 to 4 in the Hoehn and Yahr scale. We excluded individuals who were practicing some systematized

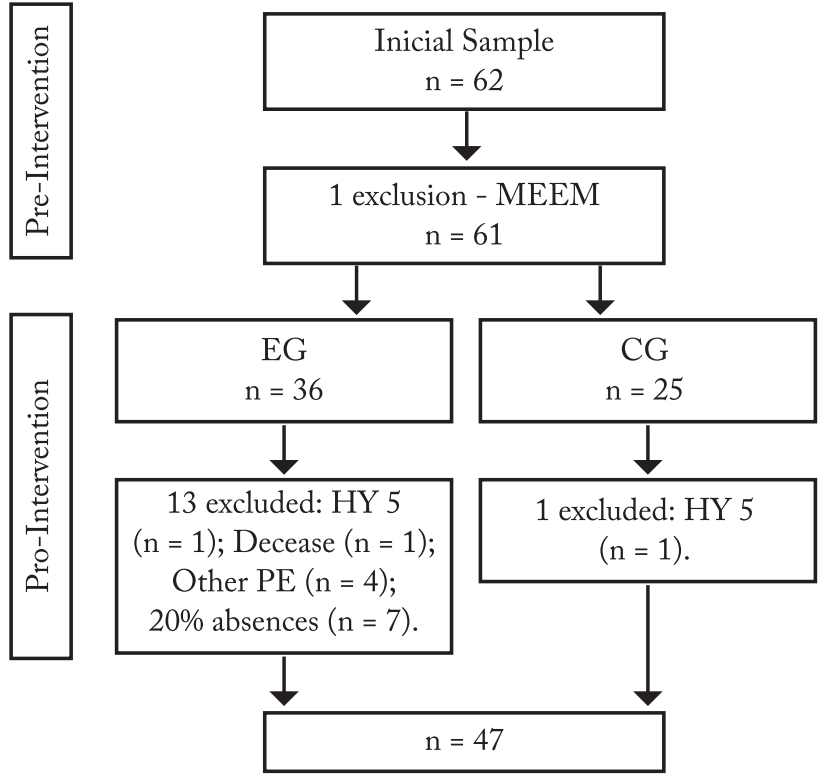

Figure 1 - Flowchart of study participant's selection (CG and EG).

physical activity; with scores in the Mini Mental State Examination lower than the reference values presented by Bertolucci et al. ${ }^{11}$ (13 points for illiterate; 18 for medium education; 26 for high education); demonstrating severe cognitive impairment; those with physical limitations, impairing daily life activities or social activities originating from sources other than $\mathrm{PD}$, which did not finish all stages of the study and those classified in Stage 5 (wheelchair) of the disease ${ }^{12}$.

The project was approved by the Ethics Committee on Human Research (CEPSH) of the UDESC under opinion No. 1,268,353 and in the Brazilian Registry of Clinical Trials (REBEC) with the indicator RBR82M4D5. Data collection was performed in the CCR by four trained researchers, who did not know to which group the individuals had gone; all participants were informed about the procedures of the study and also about the importance of classes' attendance for their health maintenance, and for the research development. After signing the informed consent, data were collected in two stages: a) Pre-intervention - which was collected in the week prior to the start of dance classes; and b) Post-intervention - occurring after the intervention of 12 weeks using the same instruments of the first collection.

The dance classes occurred according to the Brazilian samba protocol validated for individuals with $\mathrm{PD}^{13}$ and was strictly followed as reference, which proceeded as follows: occurred for 12 weeks, twice a week with one hour duration divided into warming (10'), main part 
(35') and relaxation (15'). The responsibility for the development of the activities belonged to three researchers of the area and one more Brazilian samba teacher who only participated in the application of the intervention.

For CG data collection, which occurred concurrently with the EG, the researchers went to the households of individuals who agreed to participate in the study, explained the entire research procedure and instructed them to maintain normal activities in the reference period to the intervention warning about the benefits of a physical activity program for the maintenance of their health.

For the data collection, a questionnaire was used as an interview divided into five parts:

- Mini Mental State Examination (MMSE): It was used as an exclusion criterion and the cutoff point was established from the values of Bertolucci et al. ${ }^{11}$ (13 points for illiterate; 18 for medium education; 26 for high education);

- Unified Parkinson's Disease Rating Scale (UPDRS). Divided into: mental activity, behavior and mood; activities of daily living; motor exploration and complications of therapy - scores range from zero to four; with higher values representing greater commitments caused by the disease ${ }^{14}$;

- Balance: Berg Balance Scale (BBS). It was validated in Brazil by the study carried out by Scalzo et $a . .^{15}$. The instrument assesses functional balance in 14 topics of daily living. The evaluation is done through the researcher observation in the accomplishment of activities carried out by the subject; the score for each item ranges from zero to four, totaling 56 points. Scores follow an increasing order of functional independence, namely: $\leq 20$ wheelchair user; $>20 \leq 40$ can walk but with assistance; > $40 \leq 56$ independent ${ }^{16,17}$. A higher score represents better balance.

- Quality of Life (QOL): PDQ-39 Brazilian version. The questionnaire is divided into eight dimensions: Mobility, Daily Life Activities, Emotional Well-Being, Stigma, Social Support, Cognition, Communication and Body Discomfort. The total score varies from zero (no problem) to 100 (maximum problem level), that is, a low score indicates a better perception of the QoL by the individual ${ }^{18}$;

- Perceived change: in the post-intervention period, individuals provided a global value of the perceived changes (Perception of the Magnitude of Perceived Changes) compared to the symptoms reported pre- and post-intervention. The results were used to verify the minimum difference clinically important (MDCI) based on the anchor-based method ${ }^{19}$.

The sample calculation was performed using $G$ POWER software 3.1.9.2 $2^{20}$; adopting the following parameters: a moderate effect $(0,447)^{21}$ of Brazilian samba on motor symptoms (UPDRS III), test power of 0.9 and $\alpha=0.05$ between EG and CG, based on similar dance and Parkinson's interventions ${ }^{22}$. Anticipating sample loss of 20\%, 30 subjects were included per group, reaching a total of 60 individuals.

We created a database in IBM SPSS Statistics 20.0. Descriptive statistics were performed for the knowledge of the data. Using two-way, repeated-measures analyses of variance using the Sydak correction for multiple comparisons. The compared variables included the balance, UPDRS and QV score to determine changes between pre- and post-test in both groups, and to compare the control and experimental groups at post-test. The significance level was set at 5\%.

\section{Results}

The majority of participants in both groups (EG and $\mathrm{CG}$ ) were male, had concomitant diseases of PD, and those of the circulatory system were the most cited. In relation to the subgroup of $\mathrm{PD}$, the dominant tremor was predominant. The EG individuals presented lower values $(6.1 \pm 4.4$ years $)$ than those of the CG $(6.9 \pm 6.9$ years) at the first symptom's date and higher in the diagnosis of the disease, being the EG, with approximately two years until the disease diagnosis and the CG, with one year approximately (data not shown in the table).

Concerning the total UPDRS (Figure 2), a significant difference was observed between the pre $(45 \pm 4)$ and post $(36 \pm 4)$ weeks intervention of the EG (p < 0.001 ), showing improvement in this variable, however in the CG, no significant differences were found for the total UPDRS.

Table 1 shows a significant improvement of balance only in the post-intervention periods, both in the EG $(\mathrm{p}=0.003)$ and in comparison between the groups after the twelve weeks $(p=0.010)$. Regarding QoL, only the EG showed a significant improvement after the intervention in the mobility domain $(p=0.007)$. In the post-intervention period only the EG presented significant differences for the cognitive $(p=0.025)$ and communication $(p=0.021)$ domains.

Figure 3 shows that most individuals (77\%) report- 
Table 1 - Comparison of the variables after twelve weeks intragroup and between CG and EG of subjects with PD.

\begin{tabular}{|c|c|c|c|c|c|c|c|c|c|}
\hline & & CG & & & EG & & & & \\
\hline & Pre & Post & $\mathrm{CE}$ & p-value* & Pre & Post & $\mathrm{CE}$ & p-value* & p-value\# \\
\hline Balance & $38.60 \pm 4.7$ & $37.00 \pm 4.6$ & -1.6 & 0.454 & $46.50 \pm 7.7$ & $49.90 \pm 7.0$ & 3.4 & $0.003^{* *}$ & $0.010^{* *}$ \\
\hline \multicolumn{10}{|l|}{ PDQ-39 } \\
\hline Mobility & $20.70 \pm 10.9$ & $19.40 \pm 12.5$ & 1.2 & 0.344 & $16.60 \pm 12.3$ & $12.60 \pm 12.3$ & 3.9 & $0.007^{* *}$ & 0.069 \\
\hline Daily Activity & $10.90 \pm 7.1$ & $11.20 \pm 7.7$ & -0.3 & 0.648 & $7.40 \pm 5.4$ & $7.50 \pm 6.3$ & -0.1 & 0.907 & 0.080 \\
\hline Emotional & $10.00 \pm 5.9$ & $9.80 \pm 6.1$ & 0.2 & 0.839 & $9.80 \pm 5.4$ & $8.10 \pm 5.0$ & 1.7 & 0.110 & 0.317 \\
\hline Stigma & $2.80 \pm 4.0$ & $2.70 \pm 4.1$ & 0.1 & 0.898 & $4.40 \pm 4.0$ & $3.80 \pm 3.8$ & 0.6 & 0.328 & 0.360 \\
\hline Support & $6.70 \pm 2.7$ & $5.90 \pm 3.0$ & 0.8 & 0.082 & $5.90 \pm 3.1$ & $6.50 \pm 2.2$ & -0.6 & 0.179 & 0,413 \\
\hline Cognitive & $7.80 \pm 3.7$ & $6.70 \pm 3.7$ & 1.0 & 0.191 & $5.30 \pm 4.0$ & $4.50 \pm 2.7$ & 0.7 & 0.361 & $0.025^{* *}$ \\
\hline Communication & $3.80 \pm 2.9$ & $3.70 \pm 2.9$ & 0.1 & 0.782 & $2.60 \pm 2.3$ & $1.90 \pm 2.0$ & 0.7 & 0.095 & $0.021^{* *}$ \\
\hline Discomfort & $5.40 \pm 3.1$ & $5.20 \pm 3.5$ & 0.2 & 0.582 & $5.20 \pm 2.5$ & $4.80 \pm 3.1$ & 0.3 & 0.400 & 0.698 \\
\hline Total & $68.10 \pm 26.8$ & $64.60 \pm 31.5$ & 3.5 & 0.365 & $57.20 \pm 25.2$ & $49.70 \pm 26.1$ & 7.4 & 0.063 & 0.087 \\
\hline
\end{tabular}

$\mathrm{CG}=$ Control Group; EG = Experimental Group; CE = Change Score; ${ }^{*}$-values of two-way, repeated measures analyses of variance using the Sydak correction for multiple comparisons; \#post-intervention values between groups; ** $\mathrm{p}<0,05$

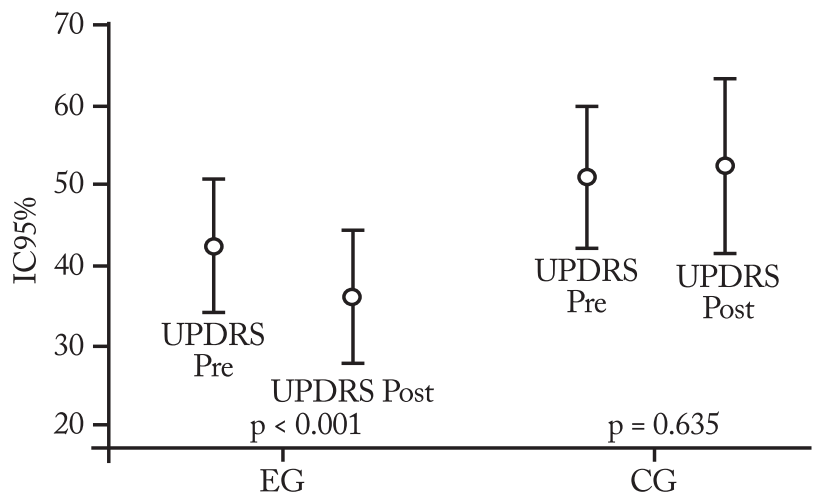

Figure 2 - Comparison between CG and EG in the pre- and post-intervention periods, according to the total UPDRS scale. Legend: IC95\% = Confidence interval; $\mathrm{P}$ = p-value; $\mathrm{EG}=$ Exercise group; $\mathrm{CG}=$ Control group.

ed improvement in their condition perception after the 12-week intervention with the Brazilian samba dance.

Figure 4 shows the perceived changes by study participants in relation to balance and total QOL. Regarding the perception of the balance change, $66.7 \%$ of the group that obtained improvement in balance, reported to perceive this change, and even the group that did not obtain improvement in the balance, $80 \%$ responded to perceive improvement. In the total QoL, in the group that achieved positive change, $100 \%$ perceived this improvement and even in the group that did not change, 50\% reported having perceived improvement and for those that the change in total QoL was negative, $77 \%$ responded to perceive improvement.

\section{Discussion}

The aim of the present study was to analyze the impact of Brazilian samba training protocol on the ba-

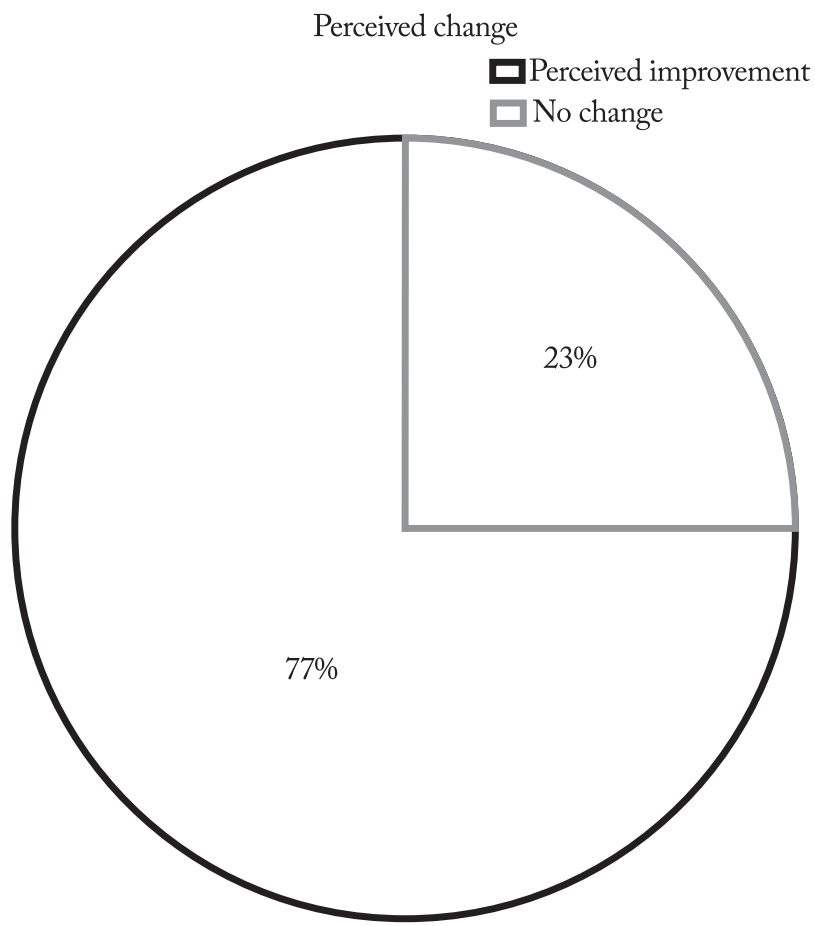

Figure 3 - Change perceived by individuals with PD who participated in the intervention

lance and QoL of individuals with PD. The results demonstrate that Brazilian samba positively influences balance and QoL of these individuals after 12 weeks of intervention. As in other studies already carried out with different dance styles ${ }^{5,6,22}$, the GE also showed a significant improvement in the total UPDRS after the intervention with Brazilian samba, it is noted that the dance classes stimulate several aspects that can influence the UPDRS, such as motor function, gait, balance, cognitive function and the improvement of the apathy 

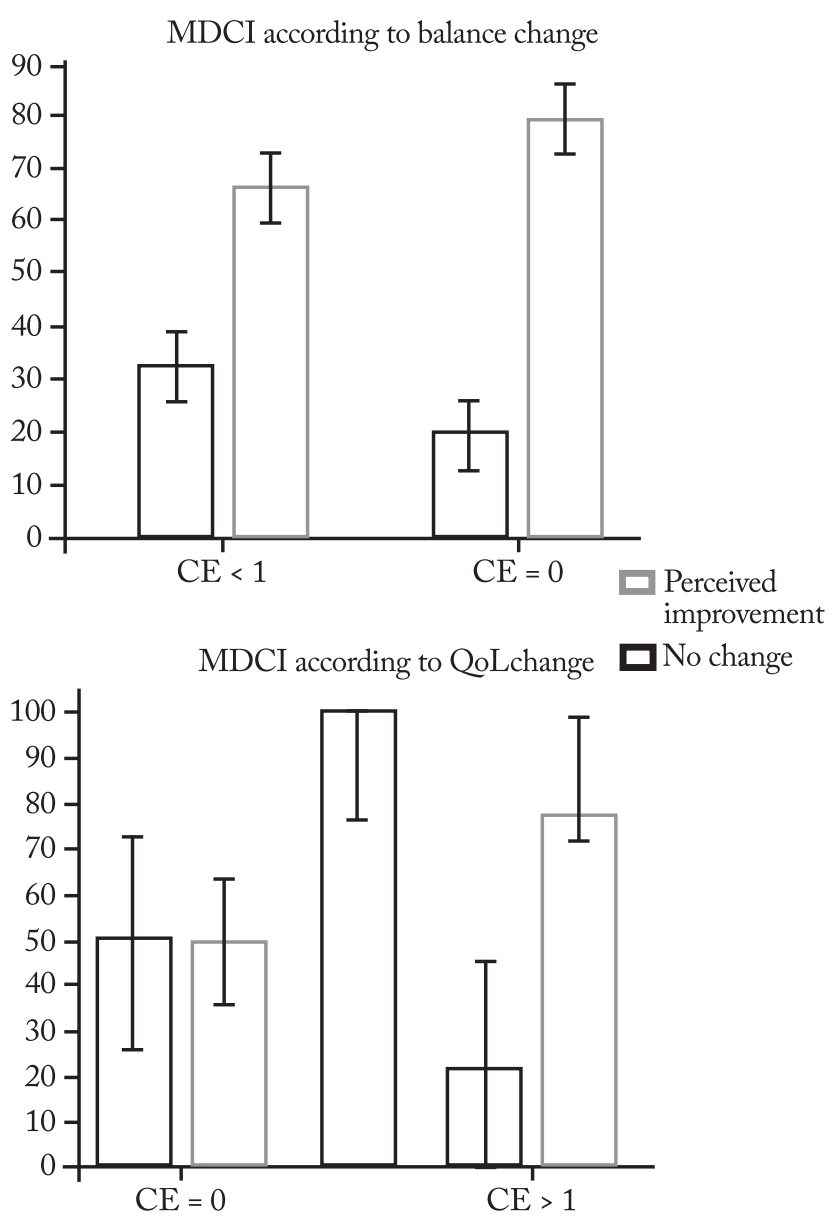

Figure 4- Perceived change in balance and total QoL by individuals with $\mathrm{PD}$.

Legend: $\mathrm{MDCI}=$ minimal clinically important difference; $\mathrm{QoL}=$ Quality of life; $\mathrm{CE}$ = change score.

of individuals with $\mathrm{PD}^{22}$.

The main outcome of this study, the balance, showed improvement in the scores after the 12 weeks of intervention in the EG also representing a difference in the final comparison with the CG - which presented worsened by 1.6 points at the end of the twelve weeks. Progress in balance was also found in the literature with ballroom dancing as those performed using the Tango by Duncan \& Earhart ${ }^{23}$ and with the Irish dance by Volpe et $\mathrm{al}^{4}$. Thus, dance seems to improve balance through the use of sensory, auditory, somatosensory information, proprioception that are crucial in the control of balance, and by activating cortical areas such as the posterior parietal cortex, premotor cortex and the area supplementary motor, providing improvement in motor skills, such as balance ${ }^{24}$. Use of Brazilian samba steps such as stops, starts, changes of direction, displacements and turns provide improvement in agility and balance ${ }^{25}$.
This result seems to have an impact on the QoL of these individuals, since one of the scores that showed the greatest changes in the EG after the intervention was the mobility domain of PDQ-39. Similar results are found in the Heiberger's ${ }^{26}$ study and show that classical ballet classes improved the mobility of individuals with PD. The study by Hackney and Earhart ${ }^{27}$ and Duncan and Earhart ${ }^{23}$, corroborate these findings and show that ballroom dance interventions have a possible impact on the mobility and balance of individuals with PD. It is believed that the intervention with ballroom dances influences the mobility of individuals with PD by proposing in their movement challenges for their balance, with alternating movements of walking and pauses in the execution of movements and their rhythmic execution ${ }^{23}$. In this case, the Brazilian samba propose these same stimuli in all the movements proposed in the protocol, with emphasis on steps such as ginga (forward and backward weight shift), lady spin (this is led by the gentleman for spinning) or even the basic step (switching in one step forward, two in place and one back) among others ${ }^{13}$.

Other two satisfactory results concerning individuals QoL, namely cognition and communication, are worth mentioning, regarding the first, an effective strategy to reduce PD impairments appears to be motor based rehabilitation for cognition training, through the movement in which selection and testing are required for movement planning and execution ${ }^{28}$, which justifies participants improvements in cognition after Brazilian samba intervention. In dance, for movement sequencing, attention is paid to music and concentration of signals, while at the same time, the next movement is planned - this seems to stimulate the activation of the basal ganglia and frontal lobe regions associated with movement, influencing short-term memory, selection and executing actions. These results corroborate with others already described in the literature ${ }^{22}$ after dance interventions for individuals with PD, strengthening the findings of the present study regarding the positive influence of Brazilian samba for them.

As communication score improvements, it is directly influenced by the interaction between the partners, teachers and the class; the positive experiences in the classes decrease the possibilities of isolation of the participants, since they find a way to communicate by mediation of music and dance, increasing the connection with other individuals ${ }^{29}$. Moreover, during classroom dance classes where there is flexibility in the steps routines, the 
communication between the partners must be clear for the correct sequence and execution of the steps ${ }^{30}$.

Increasing the QoL and balance scores were objectives achieved in this study. However, an interesting fact that must be considered is that $77 \%$ of individuals noticed and felt this improvement in balance and quality of life after the 12 weeks of intervention. Even individuals who did not show statistically significant improvement, reported a positive perception in these aspects after Brazilian samba classes. This may have occurred due to the improvement of the individuals' self-esteem, provided by artistic therapy, influencing their own way of coping, as well as living with others and with the disease ${ }^{16}$. These values reaffirm the potential of the Brazilian samba as a therapeutic tool in PD and can bring direct benefits as in the balance of these individuals as indirect as in the $\mathrm{QV}$ of these individuals. The comparisons with the CG that only performed conventional physiotherapy show that the EG presented quite satisfactory results in all the studied outcomes.

This study presents limitations. One of them is the non-randomization of the sample to the control and intervention groups. In addition, the sample loss during the research stages, ending with a smaller number than expected. In conclusion, individuals with PD who participated in the Brazilian samba intervention were influenced expressively, in comparison with those who did not participate in the intervention. These presented significant results in total UPDRS, balance and in QoL (mobility, cognition and communication); which demonstrates the efficacy of this rhythm for individuals with PD. These results make clear the importance of new studies in this area, mainly with a greater diversity of activities, making possible the condition improvements of this population in more pleasant ways for these.

\section{Conflict of interest}

The authors declare no conflict of interest.

\section{Funding}

The main investigator of this study was financed in part by the Coordenação de Aperfeiçoamento de Pessoal de Nivel Superior - Brasil (CAPES) (Finance Code 001).

\section{Author's contributions}

Fonseca LC, Moratelli J and Tillmann AC conceived of the study, initiated the study design, developed the methodology, and commented on initial drafts of the manuscript. Swarowsky A,
Guimaraes ACA, initiated the study design and commented on initial drafts of the manuscript. All authors read and approved the final manuscript.

\section{References}

1. Lee HK, Altmann LJ, McFarland N, Hass CJ. The relationship between balance confidence and control in individuals with Parkinson's disease. Parkinsonism Related Disorder. 2016;26:24-8.

2. Ferreira NC, Caetano FM, Damázio LCM. Correlação entre mobilidade funcional, equilíbrio e risco de quedas em idosos com doença de Parkinson. Rev Bras Geriatr Gerontol. 2011;5(2):74-9.

3. Zhang TY, Hu Y, Nie ZY, Jin RX, Chen F, Guan Q, et al. Effects of Tai Chi and Multimodal Exercise Training on Movement and Balance Function in Mild to Moderate Idiopathic Parkinson Disease. Am J Phys Med Rehab. 2015;94(10):921-9.

4. Volpe D, Signorini M, Marchetto A, Lynch T, Morris ME. A comparison of Irish set dancing and exercises for people with Parkinson's disease: a phase II feasibility study. BMC geriatr. 2013;13.

5. Houston S, Mcgill A. A mixed-methods study into ballet for people living with Parkinson's. Arts Health. 2013;5(2):103-19.

6. Lotzke D, Ostermam T, Bussing A. Argentine tango in Parkinson Disease- a systematic review and meta-analysis. BMC Neural. 2015;5.

7. Sharp K, Hewitt J. Dance as intervention for people with Parkinson`s Disease: a systematic review and meta-analysis. Neurosci Biohav. 2014;47:445-56.

8. McNeely ME, Mai MM, Duncan RP, Earhart GM. Differential Effects of Tango Versus Dance for PD in Parkinson Disease. Front Aging Neurosci. 2015;21(7): 239.

9. Sumec R, Filip P, Sheardová K, Bares M. Psychological benefits of nonpharmacological methods aimed for improving balance in Parkinson's disease: a systematic review. Behav Neurol. 2015.

10. Hughes AJ, Daniel SE, Kilford L, Lees AJ. Accuracy of clinical diagnosis of idiopathic Parkinson's disease: a clinic-pathological study of 100 cases. J Neurol Neurosurg Psychiatry. 1992;55(3):181-4.

11. Bertolucci PHF, Brucki SMD, Campacci SR, Juliano Y. O mini- exame do estado mental em uma população geral: impacto da escolaridade. Arq Neuropsiquiatr. 1994;52(1):1-7.

12. Hoehn MM, Yahr MD. Parkinsonism: onset, progression and mortality. Neurology. 1967;17:427-42.

13. Tillmann AC, Swaroswsky A, Andrade A, Guimarães ACA. Brazilian samba protocol for individuals with Parkinson's Disease: A clinical non-randomized study. JMIR Res Protoc. 2017;4;6(7):e129.

14. Movement Disorders Society Force on Rating Scales for Parkinson's Disease. The unifiedParkinson's disease rating scale (UPDRS): status and recommendations. Mov Disord. 2003;18(7):738-50.

15. Scalzo PL, Nova IC, Perracini MR, Sacramento DRC, Cardoso F, Ferraz HB, et al. Validation of the Brazilian version of the berg balance scale for patients with parkinson's disease. Arq Neuropsiquiatr. 2009;67(3b):831-5.

16. Miyamoto ST, Lombardi Junior I, Berg KO, Ramos LR, Natour J. Brazilian version of the Berg balance scale. Brazilian Journal of Medical and Biological Research, 2004;37:1411-21. 
17. Qutubuddin AA, et al. Validating the Berg Balance Scale for patients with Parkinson's disease: a key to rehabilitation evaluation. Archives of Physical Medicine and Rehabilitation. 2005;86:789-92.

18. Souza RG, Borges V, Silva SMCA, Ferraz HB. Quality of life scale in Parkinson's disease PDQ-39 - (Brazilian Portuguese version) to assess patients with and without levodopa motor fluctuation. Arq Neuropsiquiatr. 2007;65:787-91.

19. Gadotti IC, Vieira ER, Magee DJ. Importance and clarification of measurement properties in rehabilitation. Braz J Phys Ther. 2006;10(2):137-46.

20. Faul F, Erdfelder E, Lang AG, Buchner A.G*Power 3: a flexible statistical power analysis program for the social, behavioral, and biomedical sciences. Behav Res Methods. 2007;39(2):175-91.

21. Cohen J. Statistical power analysis for the behavioral sciences. 2a Edição. Hillsdale: Lawrence Erlbaum Associates; 1988.

22. Hashimoto H, Takabatake S, Miyaguchi $H$, Nakanishi $H$, Naitou Y. Effects of dance on motor functions, cognitive functions, and mental symptoms of Parkinson's disease: A quasi- randomized pilot trial. Complement Ther Med. 2015;23(2):210-9.

23. Duncan RP, Earhart GM. Are the effects of communitybased dance on Parkinson disease severity balance and functional mobility reduced with time? A 2 year prospective pilot study. J Altern Complement Med. 2014;20(10):757-63.

24. Lefaivre SC, Almeida QI. Can sensory attention focused exercise facilitate the utilization of proprioception for improved balance control in PD? Gait Posture. 2015;41(2):630-33.
25. Conradsson D, Löfgren N, Ståhle A, Hagströmer M, Franzén E. A novel conceptual framework for balance training in Parkinson's disease-study protocol for a randomised controlled trial. BMC Neurol. 2012;12:111.

26. Heiberger L, Maurer C, Amtage F, Mendez-Balbuena I, Schulte-Mönting J, Hepp- Reymond MC, et al. Impact of a weekly dance class on the functional mobility and on the quality of life of individuals with Parkinson's disease. Front Aging Neurosci. 2011;3:14.

27. Hackney ME, Earhart GM. Short duration, intensive tango dancing for Parkinson disease: an uncontrolled pilot study. Complement Ther Med. 2009;17(4):203-7.

28. Hackney ME, Lee HL, Battisto J, Crosson B, McGregor KM. Context-Dependent Neural Activation: Internally and Externally Guided Rhythmic Lower Limb Movement in Individuals With and Without Neurodegenerative Disease. Front Neurol. 2015;6:251.

29. Bognar S, DeFaria AM, O’Dwyer C, Pankiw E, Simic Bogler J, Teixeira S, et al. More than just dancing: experiences of people with Parkinson's disease in a therapeutic dance program. Disabil Rehabil. 2016;39(11):1073-8.

30. Dreu, MJ, Kwakkel G, Van Wegen EE. Partnered Dancing to improve mobility for people with Parkinson's disease. Front Neuroscience. 2015;11:444.

\section{Quote this article as:}

Fonseca LC, Tillmann AC, Moratelli J, Swarowsky A, Guimarães ACA. The impact of Brazilian samba on balance and quality of life of individuals with Parkinson's disease. Rev Bras Ativ Fís Saúde. 2021;26:e0194. DOI: 10.12820/rbafs.26e0194 